\title{
Relationship of a Low Ankle-brachial Index with All-cause Mortality and Cardiovascular Mortality in Chinese Patients with Metabolic Syndrome after a 6-Year Follow-up: A Chinese Prospective Cohort Study
}

\author{
Yan Cang ${ }^{1}$, Jue $\mathrm{Li}^{2}$, Yuan-min $\mathrm{Li}^{2}$, Yun Zhou ${ }^{2}$, Yue-zhou $\mathrm{Wu}^{2}$, Xian-kai $\mathrm{Li}^{1}$, \\ Chao-fan Wang ${ }^{1}$, Hai-yan Yang ${ }^{1}$ and Ya-wei $\mathrm{Xu}^{1}$
}

\begin{abstract}
Objective Peripheral arterial disease (PAD) is a common clinical manifestation of the systemic atherosclerotic process, and the ankle-brachial index (ABI) is an ideal tool to diagnose PAD. Currently, there have been few long-term follow-up studies focused on the associations of the ABI with all-cause mortality and cardiovascular disease (CVD) mortality in Chinese MetS patients. The aim of this study was to evaluate the usefulness of $\mathrm{ABI}$ to predict the prognosis of CVD in hospitalized Chinese patients with metabolic syndrome (MetS).

Methods Participants from multi-center departments were followed up from November 2004 to January 2011. The study sample actually comprised 1,266 valid participants whose age was $\geq 35$ years. Patients were separated into four groups, with an $\mathrm{ABI} \leq 0.4,0.41-0.7,0.71-0.9$ and 0.91-1.4. An $\mathrm{ABI} \leq 0.9$ was defined as $\mathrm{PAD}$, and subjects with an $\mathrm{ABI}>1.4$ were excluded because of the false negative rate. Factors related to allcause and cardiovascular mortality were observed by Cox models and the log rank test. Potential confounding variables with values of $\mathrm{p}<0.10$ were adjusted for the multivariate analysis.

Results An abnormal ABI value was strongly, independently, and inversely correlated with the all-cause and cardiovascular mortality. After adjusting for age and other covariates, Cox models revealed that an abnormal ABI value was still correlated with the all-cause mortality (relative risk/RR/=1.82, 95\% confidence inter$\mathrm{val} / \mathrm{Cl} /=1.45-2.34 \mathrm{p}<0.01)$, and CVD mortality $(\mathrm{RR}=1.88,95 \% \mathrm{CI}=1.51-2.90 \mathrm{p}<0.01)$.

Conclusion An abnormal ABI value was not only a significant and independent risk factor for CVD, but also for the survival rate in Chinese MetS patients. Routine ABI evaluation could therefore be helpful for identifying high risk patients, especially MetS patients.
\end{abstract}

Key words: ankle-brachial index, metabolic syndrome, coronary heart disease, peripheral arterial disease, prospective cohort study, risk factors, atherosclerosis

(Intern Med 51: 2847-2856, 2012)

(DOI: 10.2169/internalmedicine.51.7718)

\section{Introduction}

Atherosclerosis is a systemic pathogenenic process, which makes an important contribution to the changes related to cardiovascular disease (CVD) (1), hypertension (2), endstage renal disease (3), and peripheral arterial disease
(PAD) (4). PAD caused by atherosclerotic occlusion in the arteries of the legs is one of the most common clinical manifestations. It affects a significant number of individuals, represents an important cause of disability, and is associated with elevated cardiovascular morbidity and mortality $(4,5)$ and increased CVD risk (4-6). The ankle-brachial index (ABI) is a non-invasive, objective, easy and reproducible

${ }^{1}$ Heart, Lung and Blood Vessel Center, Tongji University School of Medicine, China and ${ }^{2}$ cKey Laboratory of Arrhythmias of Ministry of Education of China, Tongji University, China

Received for publication March 12, 2012; Accepted for publication June 18, 2012

Correspondence to Dr. Jue Li, jueli@Tongji.edu.cn 


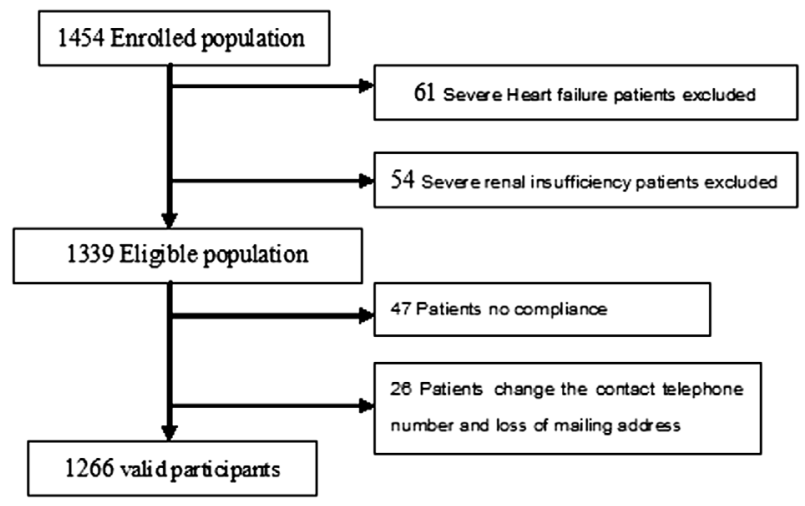

Figure 1. Study flow chart

method that can be used to detect the presence and severity of PAD, which has a 95\% sensitivity and 99\% specificity for the documentation of angiographic $\operatorname{PAD}(7,8)$. In recent years, some studies have demonstrated that a low ABI is not only associated with an increased risk of subsequent cardiovascular disease-related events, independent of Framingham risk factors $(9,10)$, but also has been examined as a tool to improve the risk prediction prospectively $(7,11-13)$.

Metabolic syndrome (MetS) is a cluster of conditions including disturbed metabolism of glucose and insulin, central obesity, dyslipidemia and hypertension. It is also associated with the subsequent development of cardiovascular disease (14). Several cohort studies have shown that MetS is a risk factor for fatal and nonfatal CVD and all-cause mortality among people with or without existing clinical cardiovascular disease, and among people with existing peripheral vascular disease (15-17).

However, there have so far been few multi-center prospective cohort studies focused on the associations of the ABI with all-cause mortality and CV mortality in Chinese PAD patients. As a result, in 2004, the China ABI Cohort Study first began to investigate the baseline characteristics of Chinese PAD patients, who were followed for up to 6 years. The aim of this study was to elucidate the relative risk factors for mortality (all-cause and CV-related), the prognostic impact for CVD in patients with MetS, and to evaluate the usefulness of the ABI to predict the prognosis of cardiovascular disease in hospitalized Chinese patients with MetS.

\section{Materials and Methods}

\section{Study population}

The study was a longitudinal cohort study. The first crosssectional survey was conducted in November 2004, in which 1,459 subjects participated. There were 61 patients suffering from severe heart failure, 54 patients with severe renal insufficiency disease and five patients with an $\mathrm{ABI}>1.40$, all of whom were excluded from the study. Therefore, 1,339 eligible participants were followed up from November 2005 to January 2011 (mean follow-up: 68.85 \pm 11.37 months).
During the follow-up period, 47 patients had missing data and 26 were non-compliant. Therefore, the study sample actually comprised 1,266 valid participants (659 men, 607 women) who were 35 years of age or older (mean age $67.8 \pm 10.6$ years) (Fig. 1). All of these hospitalized patients were consecutively enrolled from the Endocrinology or Cardiology department of eight university hospitals in Beijing and Shanghai. All subjects were under treatment because of cardiovascular diseases or endocrine diseases.

The inclusion criteria were an age of 35 years or older, and a status that conformed to the International Diabetes Federation (IDF) definition of a MetS diagnosis or patients who reported having MetS at the baseline examination. PAD was defined by an $\mathrm{ABI} \leq 0.90$ associated with stenosis of one or more lower extremity arteries. The exclusion criteria were severe heart failure or renal failure, and subjects with an ABI $>1.40$ were also excluded because these values may be falsely elevated due to severe arterial calcification $(5,8,9,18)$. In this study, there were 61 subjects with severe heart failure and 54 with severe renal failure.

We performed a subgroup analysis for the threshold level of the ABI, and chose the best cut-off value to predict the outcome according to a time-dependent ROC curve analysis (19-21). Thus, the ABI was categorized as: $\leq 0.4,0.41$ $0.70,0.71-0.90$ and $0.91-1.40$ to show the relationship with the all-cause and CVD mortality (13). All participants gave written, informed consent for participation in this study, which was approved by the ethics committee of Tongji University.

\section{Measurement of ankle and arm blood pressure}

Qualified ultrasonographers measured the ankle and brachial systolic blood pressures. Doppler ultrasound (Nicolet Vascular, Elite 100R, USA) was used to measure the systolic blood pressure (SBP) in the bilateral brachial, tibial and dorsal pedal arteries. The occluding cuffs $(55 \pm 12.5 \mathrm{~cm})$ were applied just above the malleoli to measure the ankle pressure. The ABI was measured after participants had rested in the supine position for 5 minutes. The ABI was calculated as the average of the two ankle systolic measurements divided by the average of the first two brachial readings. The lower ABI of the two legs was used as a predictor of future cardiovascular events.

\section{Diagnosis of PAD}

As recommended by the American College of Cardiology (ACC) and the American Heart Association (AHA) guidelines, and in agreement with recently reported studies of PAD, we adopted an ABI $\leq 0.90$ as indicative of a diagnosis of $\operatorname{PAD}(18,22,23)$. Therefore, an $\mathrm{ABI} \leq 0.90$ in either leg was considered to be a low ABI, and such participants were diagnosed as having PAD. Moreover, this cut-off point was also used in the Strong Heart Study (SHS), in which the upper limit of normal ABI did not exceed 1.40 (21). Therefore, the ABI range from 0.91 to 1.40 was considered the normal range among all participants. 


\section{Diagnosis of diabetes}

The diagnosis of diabetes was based on a previous history of diabetes treated with or without drug therapy or fulfillment of the WHO 1999 criteria (24). The participants were classified into categories of glucose intolerance or diabetes at baseline and follow-up based on their fasting plasma glucose (FPG) and $2 \mathrm{~h}$ post-glucose load (2 h PG), impaired fasting glucose (IFG) (FPG 6.1-6.9 mmol/L), impaired glucose tolerance (IGT) (2 h PG 7.8-11.0 mmol/L), impaired glucose regulation (IGR) (FPG 6.1-6.9 mmol/L and/or $2 \mathrm{~h}$ PG 7.8-11.0 mmol/L), and diabetes (FPG $\geq 7.0 \mathrm{mmol} / \mathrm{L}$ or 2 h PG $\geq 11.1 \mathrm{mmol} / \mathrm{L}$ ).

\section{Definition of MetS}

MetS has been defined by the World Health Organization (WHO), European Group for the Study of Insulin Resistance (EGIR), ACE, the International Diabetes Federation (IDF), and the National Cholesterol Education Program and AHA (updated NCEP) criteria. The current five definitions were associated with different levels of increased risk for either incident coronary-heart disease (CHD) alone or CHD combined with diabetes. The results from Chinese population prospective studies have shown that the IDF definition appears to be more effective at predicting incident diabetes, total incident CHD and more compromise to predict the prognosis of cardiovascular disease in Chinese subjects $(25,26)$. Accordingly, our study adopted the IDF criteria as the definition of MetS.

The IDF definition requires central obesity as a mandatory component for a diagnosis of the MetS, using ethnicityspecific values plus any two of the following four components: (1) triglycerides, $1.7 \mathrm{mmol} / \mathrm{L}$; (2) HDL cholesterol, < $1.03 \mathrm{mmol} / \mathrm{L}$ in men and $<1.29 \mathrm{mmol} / \mathrm{L}$ in women; (3) systolic and/or diastolic blood pressure, $\geq 130 / 85 \mathrm{mmHg}$, or treatment of previously diagnosed hypertension; (4) FPG, $5.6 \mathrm{mmol} / \mathrm{L}$ or previously diagnosed type 2 diabetes. The recommended standards of obesity for Asians were used according to the IDF definitions (BMI $\geq 25$; waist circumference $\geq 90 \mathrm{~cm}$ for men and $\geq 80 \mathrm{~cm}$ for women).

\section{Cardiovascular events}

Hospitalized myocardial infarction was classified as definite or probable based on chest pain symptoms, cardiac enzyme levels, and electrocardiographic findings, or angioplasty (27). CHD was determined to be present if there was (1) electrocardiographic (ECG) evidence of a prior myocardial infarction, (2) prior coronary artery bypass surgery or angioplasty, (3) coronary angiography showed coronary heart disease, (4) symptoms of angina were present and ECG revealed myocardial ischemia or laboratory tests showed increased cardiac enzymes and excluded other types of disease, (5) a self-reported history of a physiciandiagnosed heart attack (28). CHD death was classified as "definite" based on chest pain symptoms, hospital records, and medical history. A stroke event was classified as definite or probable based on 1) sudden onset neurological symptoms \{one major (e.g., aphasia or hemiparesis) or two minor (e.g., diplopia or dysarthria) $\}$ that lasted $>24$ hours or caused death within 24 hours (2) with no evidence of any other pathology that might have mimicked stroke (28).

\section{Baseline measurements}

Variables were obtained for all patients, which included daily habits, medical histories, and blood samples. These samples were measured for the total cholesterol (TC), triglycerides (TG), high-density lipoprotein (HDL) cholesterol, low-density lipoprotein (LDL), serum urea nitrogen (SUN), serum creatinine $(\mathrm{Cr})$, serum uric acid (UA), glucose and FPG levels. Blood samples were drawn from an antecubital vein with a 19-gauge needle in a vacutainer system, and the serum concentrations of these factors were assessed with commercially available kits. The glomerular filtration rate (GFR) was calculated as GFR $\left(\mathrm{mL} / \mathrm{min} / 1.73 \mathrm{~m}^{2}\right)=186 \times$ creatinine $^{-1.154} \times \operatorname{age}^{-0.203}$ and GFR $\left(\mathrm{mL} / \mathrm{min} / 1.73 \mathrm{~m}^{2}\right)=142 \times$ creatinine $e^{-1.154} \times$ age $^{-0.203}$ in males and females, respectively. Glucose was measured on a Hitachi 717 analyzer (Roche) using enzymatic reagents from Roche. The presence of symptomatic peripheral arterial disease was evaluated by the Rose questionnaire (29). A previous myocardial infarction or ischemic stroke was documented by hospital records. Physical examination data included the body-mass index (BMI), blood pressure and ABI. Severe congestive heart failure was defined as a cardiac functional classification of three or higher as determined by the New York Heart Association (NYHA) criteria. Severe renal failure was defined as an estimated glomerular filtration rate $<60 \mathrm{~mL} / \mathrm{min} / 1.73 \mathrm{~m}^{2}$.

\section{Risk factors}

The diagnostic criteria for hypertension was receiving antihypertensive medication or a systolic blood pressure $\geq 140$ $\mathrm{mmHg}$ or diastolic blood pressure $\geq 90 \mathrm{mmHg}(1 \mathrm{mmHg}$ $=133 \mathrm{kPa}$ ), as well as having both. The criterion for hypertension refers to patients who had single hypertension disease.

Similarly, the criterion for dyslipidemia also refers to patients who have single dyslipidemia disease. The definition of dyslipidemia is that there are abnormalities in the serum levels of lipids, including overproduction or deficiency. Abnormal serum lipid profiles may include high total cholesterol, high triglycerides, low high density lipoprotein cholesterol, and elevated low density lipoprotein cholesterol.

A questionnaire was designed to collect information about the general patient characteristics, diagnosis, medical history and family history, and previous and current medical treatment, and a biochemical examination was performed in all participants. Besides the history and duration of hypertension, cardiovascular heart disease, chronic renal disease, stroke, dyslipidemia and smoking, other potential risk factors were also recorded in this questionnaire, including the patient age, gender, race and BMI. 


\section{Follow-up methods}

For follow-up, the patients were contacted by physicians of the ABI cohort study at annual intervals. Outcomes were obtained during annual phone interviews, six yearly followup examinations, hospital records, and death records. The primary clinical event endpoints of this study were the allcause mortality and cardiovascular disease mortality, and the secondary endpoints examined were peripheral arterial disease incidents, including the development of CVD. The follow-up time was considered to be the number of years since the initial baseline visit. For patients who had more than one event, only the first clinical event was considered for the analysis.

\section{Assessment of cardiovascular events and identifica- tion of death from all causes and from CVD}

Cardiovascular events included cardiac (non-fatal myocardial infarction, unstable angina, and coronary revascularization procedures), cerebrovascular (fatal and non-fatal ischemic stroke), and peripheral (patients with no previous history of PAD and normal ABI during the follow-up time confirmed by the presence of critical limb ischemia or revascularization procedures performed on the lower limbs) events. The exclusion criteria were a stable ( $>6$ months) revascularization procedure for $\mathrm{CAD}$ or $\mathrm{PAD}$, stable $(>6$ months) angina, myocardial infarction or stroke.

In this study, the cardiovascular deaths included cardiac and cerebrovascular events leading to death. The medical records and death certificates of all patients who had an event were obtained and validated by a cardiologist or endocrinologist. Death was confirmed from hospital records or by contact with the participants' families. All materials were reviewed independently by a physician of the ABI cohort study to confirm the cause of death.

\section{Statistical analysis}

All case record data were entered into two Epidata 3.02 (EpiData Association, Odense, Denmark) databases by different people. Data were analyzed using the software program SPSS13.0 (Chicago, IL, USA). Continuous variables are expressed as the means $\pm \mathrm{SD}$, and categorical variables as percentages. The differences in continuous and categorical variables were determined by an independent samples ANOVA (analysis of variance) and the chi-square test, as appropriate. The Kruskal-Wallis test was used for nonnormally distributed continuous variables. A $\mathrm{p}$ value $<0.05$ was considered to be statistically significant. Due to their skewed distribution, the TC, TG, HDL-C, LDL-C, SUN, Cr, and UA were logarithm-transformed $(\log )$ in the analyses. The all-cause and CVD-related crude deaths were examined by ABI stratification. Cumulative event rates were estimated with Kaplan-Meier survival curves, and probability values were calculated with the log-rank test. The Cox proportional hazard analyses were performed to test the association between the ABI and deaths from all-causes or CVD. A Cox regression model was adjusted for potential confounders, including age, gender, duration of hypertension, smoking, history of dyslipidemia, chronic renal insufficiency, diabetes mellitus, PAD, myocardial infarction, ischemic stroke, hypertension, ABI and the level of serum uric acid. Potential confounding variables with a value of $\mathrm{p}<0.10$ were adjusted for the multivariate analysis.

\section{Results}

\section{Baseline characteristics}

A total of 1,339 eligible participants with available baseline data were enrolled in this cohort. All patients were under treatment for secondary prevention. Among the study population, 26 individuals had missing follow-up data because they changed their telephone number or address during the follow-up period. Another 47 patients were noncompliant. Therefore, the study sample actually comprised 1,266 valid participants (659 men, 607 women) who were 35 years of age or older (mean age $67.8 \pm 10.6$ years). The average follow-up was 58 months (range: 16 to 38 months) in this study. The missing participants did not significantly affect the major results.

We found that the PAD prevalence in these patients was $24.4 \%$. The average ABI for the entire population was $1.15 \pm$ 0.12 (mean $\pm \mathrm{SD}$ ). Table 1 presents the baseline characteristics of our patients. The patients were divided into four groups according to the ABI value: $\leq 0.4,0.41-0.70,0.71$ 0.90 and $0.91-1.40$. Among the numerous variables examined, our research revealed that the abnormal ABI group comprised older patients, and that these patients had a higher SBP level, SUN concentration, serum uric acid concentration, ischemic stroke history, myocardial infarction history, longer duration of hypertension, DM and smoking rates. Of note, for these above-mentioned variables, the risk factor levels all increased with decreasing ABI values. Although there was an increasing tendency for the diastolic blood pressure, BMI, dyslipidemia history, dyslipidemia duration, and history of chronic renal insufficiency with decreasing $\mathrm{ABI}$ levels, there were no statistically significant differences between the low ABI group and normal ABI group. The lipid levels also were not significantly different between the low ABI group and normal ABI group.

\section{All-cause and CVD-related mortality rates}

As illustrated in Fig. 2, the all-cause mortality in the groups with an $\mathrm{ABI} \leq 0.4,0.4<\mathrm{ABI} \leq 0.7,0.7<\mathrm{ABI} \leq 0.9$, $0.9<\mathrm{ABI} \leq 1.4$ were 37 (50.0\%), $62(40.0 \%), 57(27.1 \%)$ and $147(17.8 \%)$, respectively. This showed the same trend as the CVD mortality rates, the values of which were 30 $(40.5 \%), 34(21.9 \%), 27(12.9 \%)$ and $66(8.0 \%)$, respectively. From Fig. 2, it can also be seen that the all-cause and CVD-related mortality rates were significantly higher in the low ABI group than the normal ABI group. There was a significant increasing tendency in all-cause mortality and car- 
Intern Med 51: 2847-2856, 2012 DOI: 10.2169/internalmedicine.51.7718

Table 1. Comparison of Baseline Characteristics according ABI in Patients with Metabolic Syndrome

\begin{tabular}{|c|c|c|c|c|c|}
\hline Number of subjects (\%) & $\mathrm{ABI} \leq 0.4$ & $0.4<\mathrm{ABI} \leq 0.7$ & $0.7<\mathrm{AB} \mid \leq 0.9$ & $0.9<\mathrm{AB} \mid \leq 1.4$ & $p$ value \\
\hline Gender & & & & & 0.129 \\
\hline Male & $7(33.3 \%)$ & $54(45.4 \%)$ & $91(54.2 \%)$ & $507(52.9 \%)$ & \\
\hline Female & $14(66.7 \%)$ & $65(54.6 \%)$ & $77(45.8 \%)$ & $451(47.1 \%)$ & \\
\hline age(year) & $74.00 \pm 9.63$ & $71.97 \pm 8.57$ & $68.92 \pm 8.00$ & $63.49 \pm 7.55$ & $<0.001$ \\
\hline Smoking History(\%) & & & & & $<0.001$ \\
\hline Yes & $10(47.6 \%)$ & $51(42.9 \%)$ & $69(41.1 \%)$ & $358(37.4 \%)$ & \\
\hline No & $11(52.4 \%)$ & $68(57.1 \%)$ & $99(58.9 \%)$ & $600(62.6 \%)$ & \\
\hline Smoking duration (years) & $15.97 \pm 2.09$ & $13.14 \pm 1.81$ & $10.95 \pm 1.59$ & $8.57 \pm 1.50$ & $<0.001$ \\
\hline Hypertension History(\%) & & & & & 0.005 \\
\hline Yes & $18(85.7 \%)$ & $108(90.8 \%)$ & $147(87.5 \%)$ & $765(79.9 \%)$ & \\
\hline No & $3(14.3 \%)$ & $11(9.2 \%)$ & $21(12.5 \%)$ & $193(20.1 \%)$ & \\
\hline Hypertension duration (years) & $15.10 \pm 1.34$ & $14.57 \pm 1.34$ & $14.56 \pm 1.31$ & $10.42 \pm 1.13$ & $<0.001$ \\
\hline $\mathrm{SBP}(\mathrm{mmHg})$ & $152.05 \pm 24.37$ & $149.95 \pm 22.68$ & $143.28 \pm 21.67$ & $141.21 \pm 21.15$ & $<0.001$ \\
\hline $\mathrm{DBP}(\mathrm{mmHg})$ & $84.76 \pm 14.42$ & $82.76 \pm 13.93$ & $82.49 \pm 12.54$ & $82.24 \pm 12.42$ & 0.849 \\
\hline BMI (kg/m2) & $27.99 \pm 2.50$ & $27.80 \pm 2.44$ & $27.79 \pm 2.35$ & $27.70 \pm 2.32$ & 0.445 \\
\hline ischemic stroke History(\%) & & & & & $<0.001$ \\
\hline Yes & $13(61.9 \%)$ & $60(50.4 \%)$ & $73(43.5 \%)$ & $257(26.8 \%)$ & \\
\hline No & $8(38.1 \%)$ & $59(49.6 \%)$ & $95(56.5 \%)$ & $701(73.2 \%)$ & \\
\hline myocardial infarction History(\%) & & & & & 0.001 \\
\hline Yes & $7(33.3 \%)$ & $29(24.4 \%)$ & $37(22.0 \%)$ & $138(14.4 \%)$ & \\
\hline No & $14(66.7 \%)$ & $90(75.6 \%)$ & $131(78.0 \%)$ & $820(85.6 \%)$ & \\
\hline PAD History(\%) & & & & & 0.003 \\
\hline Yes & $6(28.6 \%)$ & $32(26.8 \%)$ & $42(25.0 \%)$ & $168(17.5 \%)$ & \\
\hline No & $15(71.4 \%)$ & $87(73.2 \%)$ & $126(75.0 \%)$ & $790(82.5 \%)$ & \\
\hline Diabetes History(\%) & & & & & $<0.001$ \\
\hline Yes & $14(66.7 \%)$ & $66(55.5 \%)$ & $85(50.6 \%)$ & $382(39.9 \%)$ & \\
\hline No & $7(33.3 \%)$ & $53(44.5 \%)$ & $83(49.4 \%)$ & $576(60.1 \%)$ & \\
\hline DM duration (years) & $6.81 \pm 1.01$ & $6.08 \pm 0.82$ & $4.11 \pm 0.63$ & $2.80 \pm 0.53$ & $<0.001$ \\
\hline dyslipidemia history(\%) & & & & & 0.103 \\
\hline Yes & $10(47.6 \%)$ & $51(42.9 \%)$ & $71(42.3 \%)$ & $421(43.9 \%)$ & \\
\hline No & $11(52.4 \%)$ & $68(57.1 \%)$ & $97(57.7 \%)$ & $537(56.1 \%)$ & \\
\hline dyslipidemia duration (years) & $5.05 \pm 1.82$ & $5.04 \pm 1.73$ & $4.99 \pm 1.69$ & $4.98 \pm 1.67$ & 0.470 \\
\hline chronic renal insufficiency History $(\%)$ & & & & & 0.027 \\
\hline Yes & $5(23.8 \%)$ & $18(15.1 \%)$ & $22(13.1 \%)$ & $88(9.1 \%)$ & \\
\hline No & $16(76.2 \%)$ & $101(84.9 \%)$ & $146(86.9 \%)$ & $870(90.9 \%)$ & \\
\hline $\mathrm{TC}(\mathrm{mmol} / \mathrm{L})$ & $5.12 \pm 0.74$ & $4.96 \pm 0.74$ & $4.96 \pm 0.64$ & $4.93 \pm 0.61$ & 0.400 \\
\hline $\mathrm{TG}(\mathrm{mmol} / \mathrm{L})$ & $2.04 \pm 0.13$ & $1.99 \pm 0.12$ & $1.99 \pm 0.12$ & $1.98 \pm 0.11$ & 0.900 \\
\hline $\mathrm{HDL}-\mathrm{C}(\mathrm{mmol} / \mathrm{L})$ & $1.16 \pm 0.39$ & $1.14 \pm 0.38$ & $1.14 \pm 0.37$ & $1.13 \pm 0.35$ & 0.420 \\
\hline LDL-C (mmol/L) & $2.91 \pm 0.91$ & $2.83 \pm 0.90$ & $2.81 \pm 0.89$ & $2.81 \pm 0.88$ & 0.300 \\
\hline SUN (mmol/L) & $6.71 \pm 0.37$ & $6.64 \pm 0.32$ & $6.64 \pm 0.31$ & $6.55 \pm 0.28$ & 0.710 \\
\hline $\mathrm{Cr}$ (umol/L) & $104.02 \pm 5.90$ & $103.75 \pm 5.04$ & $102.99 \pm 5.35$ & $102.91 \pm 5.32$ & 0.660 \\
\hline UA (umol/L) & $370.57 \pm 11.52$ & $367.92 \pm 11.24$ & $364.94 \pm 11.20$ & $351.50 \pm 11.01$ & 0.050 \\
\hline $\mathrm{FPG}(\mathrm{mmol} / \mathrm{L})$ & $6.9 \pm 0.33$ & $6.8 \pm 0.28$ & $6.75 \pm 0.28$ & $6.75 \pm 0.22$ & 0.510 \\
\hline
\end{tabular}

systolic blood pressure (SBP ), diastolic blood pressure (DBP ), BMI(body mass index.), Total cholesterol (TC), Triglycerides(TG), Fasting plasma glucose( FPG), high density lipoprotein(HDL-C), low density lipoprotein (LDL-C), serum urea nitrogen(SUN), serum creatinine(Cr), serum uric acid(UA), diabetes mellitus( DM), myocardial infarction ( MI)

diovascular mortality with decreasing ABI levels. Further- of follow-up $(\mathrm{p}<0.001)$. more, patients whose ABI values were lower than 0.4 had the highest all-cause and CVD mortality after the six years 


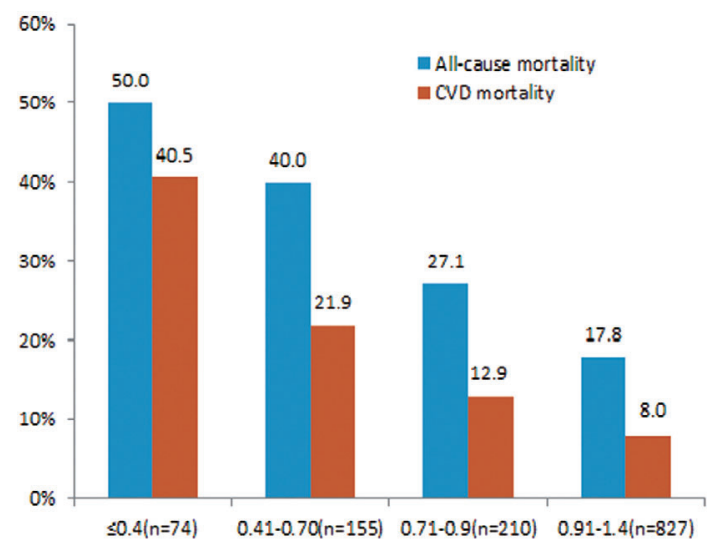

Figure 2. All-cause and CVD mortality according to ABI in the ABI Cohort Study during 6-years follow-up $(n=1,266)$.

\section{Survival analysis of the $A B I$ groups}

Fig. 3A and B illustrate the relationships between the four ABI categories of all-cause mortality and CVD mortality, respectively. The Kaplan-Meier survival curves showed that the survival rate decreases with the decrease in the ABI, not only for the all-cause mortality (Fig. 3A) but also for the CVD-related mortality (Fig. 3B). The subgroup with ABI values lower than 0.4 had the highest all-cause and CVD mortality.

\section{Mortality risk stratification according to the different $A B I$}

Fig. 4A and $\mathrm{B}$ show the adjusted relative risk (RR) of mortality according to the different ABI groups. Compared with the normal ABI group, after adjusting for gender, age, hypertension, stroke, MI history, smoking, chronic renal disease, UA and PAD history, dyslipidemia history, diabetes mellitus history, and ABI, the Cox regression models revealed that patients in the $\mathrm{ABI}<0.4$ group were about two times $(1.82,95 \%$ CI: $1.45-2.34)$ as likely to die as those with an ABI in the range of 0.91-1.4; patients with an ABI $<0.4$ were also about two times (1.88, 95\% CI: $1.51-2.90)$ more likely to die of CVD than those with an ABI in the range of 0.91-1.4. Fig. 4A and $\mathrm{B}$ also present the RR of allcause mortality and CVD mortality in the patients in the 0.4 $<\mathrm{ABI} \leq 0.7$ and $0.7<\mathrm{ABI} \leq 0.9$ groups compared to the normal ABI group, which were $(1.71,95 \%$ CI: $1.34-2.04)$ and (1.61, 95\% CI: $1.30-1.95)$ and $(1.64,95 \%$ CI: 1.31-2.17) and (1.52, 95\% CI: 1.23-2.03), respectively. The prevalence of $\mathrm{CAD}$ and stroke also tended to increase with decreasing ABI levels.

\section{Other factors predicting a risk of mortality}

As shown in Table 2, the Cox regression models also demonstrated that there are other risk factors for mortality, including gender, age, hypertension, stroke, MI history, PAD history, dyslipidemia history, smoking and UA in the abnormal ABI group. It should be noted that among these risk factors, age, hypertension, stroke and MI history were the most important.

\section{Discussion}

Epidemiological studies have revealed that MetS is an independent risk factor for CVD and all-cause mortality. Meanwhile, meta-analyses of many large observational studies with long-term follow-up have reported that the ABI is associated with coronary heart events, independent of traditional Framingham variables (30), and an abnormal ABI is accepted in current guidelines as a CHD equivalent (31). The use of a low ABI for the purposes of cardiovascular risk assessment has been recommended by AHA and ACC (32), and other groups (33-35). However, due to population and ethnic differences, most of the clinical data came from studies in Western countries, and there has been limited data from multi-center prospective cohort studies focused on Chinese PAD patients with MetS. Moreover, abnormal ABI values are associated with high blood pressure, lipid abnormalities or abnormal glucose metabolism, and these factors all individually play an important role in the occurrence and development of cardiovascular disease. It has thus been unclear whether a low ABI value is an independent risk factor for CVD in Chinese patients.

At present, there are five different definitions of MetS. According to Chinese population studies' the IDF criteria were more effective to predict the prognosis of CVD in Chinese subjects than the other criteria $(25,26)$, so we adopted the IDF criteria to define MetS in our study $(25,26)$. This study demonstrated that the prevalence of PAD in hospitalized Chinese patients with MetS was $24.4 \%$, which is significantly higher than our previous study, which demonstrated a rate of $16.7 \%$ in DM patients. This finding reveals that MetS patients have a higher prevalence of PAD. When the participants were divided into the four ABI subgroups, the baseline characteristics showed an increasing tendency in the prevalence of hypertension, ischemic stroke and coronary artery disease with the decrease in the ABI.

Therefore, an obvious question raised in this report was whether the low $\mathrm{ABI}$ is an independent risk factor for the incidence of CVD and where it is associated with a decrease in the survival rate in the Chinese MetS population. The data from our six-year follow-up study has confirmed that the patients with an $\mathrm{ABI} \leq 0.9$ had a higher all-cause mortality and CVD-related mortality. The mortality decreased with the increases in the ABI, not only the all-cause mortality, but also the CVD-related mortality. The Kaplan-Meier survival curves for our study also showed the same trend, with the survival rate decreasing significantly with the decrease in the ABI. After further adjusting for potential confounders, a Cox regression model analysis revealed that an abnormal ABI value was strongly, independently, and inversely correlated with the all-cause mortality $(\mathrm{RR}=1.82,95 \%$ CI: 1.45 2.34, $\mathrm{p}<0.01$ ), and cardiovascular disease mortality ( $\mathrm{RR}=$ 1.88, 95\% CI: 1.51-2.90, $\mathrm{p}<0.01)$. Recently, McKenna et al. reported that the 5 -year mortality was $50 \%$ in patients with 
A

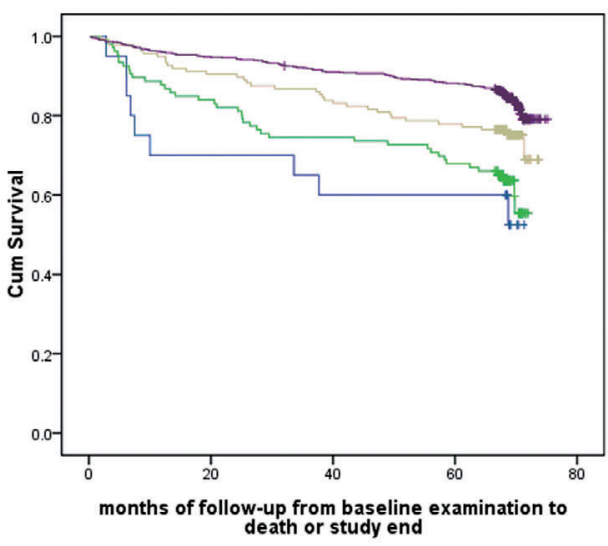

B Survival Functions

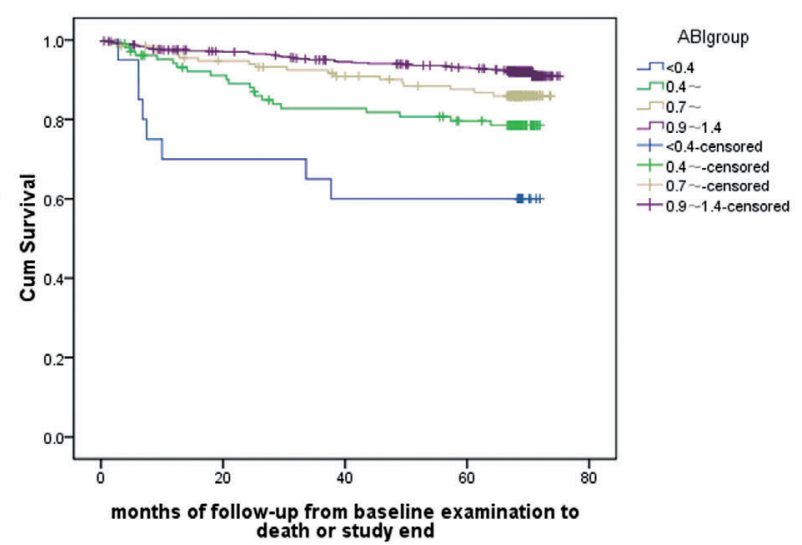

Figure 3. Time course to death from all causes (A) and CVD (B) in the ABI Cohort Study during 6-years follow-up.

A

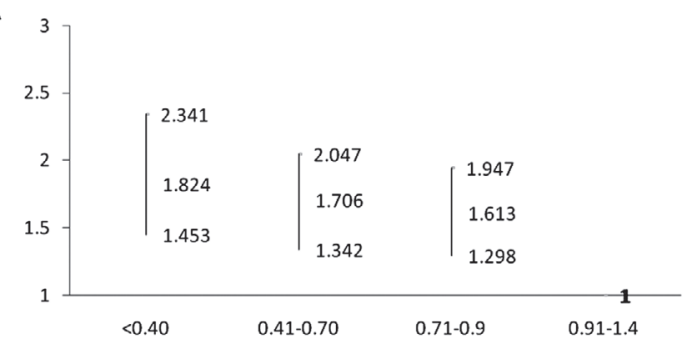

B

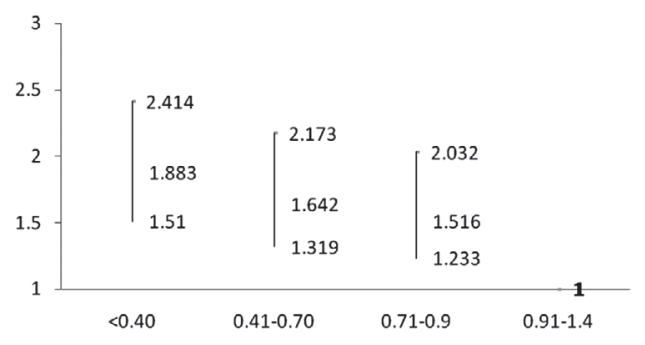

Figure 4. Adjusted relative risks for (A) all-cause mortality and (B) CVD mortality by baseline ABI in the ABI Cohort Study during 6-years follow-up.

an ABI of 0.4 and $30 \%$ in patients with an ABI of 0.70 (8). The study by Sikkink et al. showed that the cumulative survival after 5 years was $63 \%$ for patients with an ABI of $0.50,71 \%$ for patients with an ABI of 0.50-0.69 and $91 \%$ for those with an ABI of 0.70-0.89 (9). A meta-analysis from the ABI Collaboration, which included 16 studies and 48,334 participants, subdivided the ABI into 10 categories compared with a reference range of 1.11-1.20 and examined the total mortality and CV mortality (30). In this metaanalysis, the 10-year cardiovascular mortality in men with a low ABI was $18.7 \%$, but was only $4.4 \%$ for those with a normal ABI, and almost as large a difference was present in women (30). The hazard ratios were increased after adjusting for the Framingham Risk Score, and a low ABI doubled the 10-year total mortality, cardiovascular mortality, and major coronary event rates in each FRS category (30). Last year, Murphy et al. reported a 10-year follow-up study which investigated 11,594 individuals, and they revealed that an abnormal ABI is very common, occurring in $13-32 \%$ of patients (36).

Based on the above-mentioned studies, it can be concluded that patients with a low ABI value have a higher mortality rate compared with those who have a normal ABI value. Our study also confirmed that these epidemiological findings were also true for Chinese patients. All of the data showed that a low ABI value was not only a significant and independent risk factor for cardiovascular disease, but also an independent predictor of a decreased survival rate, especially among the patients with MetS.

Another obvious question raised by this study is how the low ABI is associated with the incidence of CVD and a decreased survival rate in the MetS population. Simply stated, the abnormal metabolic state directly contributes to the development of atherosclerosis. The proatherogenic changes include increases in vascular inflammation and alterations in multiple cell types (37). Most patients with MetS and PAD demonstrate generalized endothelial cell dysfunction. The effects of endothelial cell dysfunction increase the arterial susceptibility to atherosclerosis. The CRP levels are abnormally elevated in patients with impaired glucose tolerance (37). Elevated levels of C-reactive protein (CRP) are strongly associated with the development of PAD (38). Besides reducing the NO concentrations, MetS also increases the production of vasoconstrictors, such as endothelin-1 (38). Furthermore, platelet aggregation is enhanced in MetS patients (38). Therefore, alterations in metabolism increase the tendency toward coagulation, and coupled with impaired fibrinolysis, this contributes to enhance thrombotic potential (39). In brief, multiple atherosclerosis factors lead to CHD and PAD, and it is a systemic process $(38,39)$.

Our study also demonstrated that age, hypertension, ischemic stroke, myocardial infarction, PAD and smoking 
Table 2. Cox Regression Adjusting Impact Multivariate of CVD Mortality in Patients with Metabolic Syndrome

\begin{tabular}{|c|c|c|c|c|c|c|}
\hline \multirow{2}{*}{ Baseline characteristic } & \multirow{2}{*}{$\beta$} & \multirow{2}{*}{ Wald } & \multirow{2}{*}{$\mathbf{R R}$} & \multicolumn{2}{|c|}{$95 \% \mathrm{Cl}$} & \multirow{2}{*}{$\mathbf{p}$} \\
\hline & & & & low & high & \\
\hline gender (female) & 0.235 & 13.783 & 1.035 & 1.023 & 1.047 & $<0.001$ \\
\hline age (year) & 0.523 & 52.414 & 1.688 & 1.258 & 2.264 & $<0.001$ \\
\hline Smoking & 0.436 & 41.011 & 1.547 & 1.164 & 2.056 & $<0.001$ \\
\hline Chronic renal insufficiency History & 0.283 & 25.370 & 1.327 & 0.983 & 1.803 & 0.055 \\
\hline dyslipidemia History & 0.237 & 14.140 & 1.034 & 0.994 & 1.263 & 0.106 \\
\hline Diabetes mellitus History & 0.333 & 26.916 & 1.395 & 1.167 & 2.296 & $<0.001$ \\
\hline PAD History & 0.244 & 20.187 & 1.155 & 1.096 & 1.824 & $<0.001$ \\
\hline serum uric acid & 0.221 & 12.751 & 1.013 & 1.009 & 1.176 & 0.015 \\
\hline Hypertension & 0.658 & 55.627 & 1.890 & 1.636 & 1.939 & $<0.001$ \\
\hline ischemic stroke & 0.688 & 58.429 & 2.093 & 1.781 & 2.991 & $<0.001$ \\
\hline myocardial infarction & 0.662 & 57.751 & 1.907 & 1.581 & 2.653 & $<0.001$ \\
\hline$A B I$ & 0.649 & 54.237 & 1.883 & 1.514 & 2.414 & $<0.001$ \\
\hline
\end{tabular}

Body mass index (BMI), cardiovascular disease ( CVD), Peripheral arterial disease (PAD), ankle brachial index $(A B I)$, relative risk $(R R)$. Beta $(\beta)$ is the coefficient of item $X$ in equation. Wald is variable value of Wald statistic test.

are strongly associated with the development of CVD and the total mortality. Of note, our study enrolled only subjects with MetS, therefore, our findings did not reveal that dyslipidemia and FPG have a statistically significant relationship.

In conclusion, our present findings further support that the $\mathrm{ABI}$ is an ideal tool for predicting mortality in MetS patients. The ABI is non-invasive, objective, easy and reproducible method that can be used to detect PAD. In clinical practice, ABI measurement should serve as a regular method for PAD screening. Meanwhile, more intensive management and aggressive treatment of vascular risk factors should be encouraged in PAD populations, especially in subjects with PAD combined with MetS

\section{Study limitations and strengths}

First, in order to ensure that the patient group was as homogenous as possible, our study adopted the IDF criteria to define MetS. Therefore, our results cannot be extended to the entire MetS population as reported in studies performed in other populations. Second, since the followed-up patients were contacted by annual phone interviews, the outcomes may have information bias. In addition, as some patients had inferior compliance, a withdrawal bias may be present in our study. Finally, in comparison with foreign prospective cohort studies, the duration of the follow-up in our study was relatively short. Hence, the data from this study are not comprehensive.

The strengths of our study include its prospective design and reliable assessment of cardiovascular events. Second, this report is the only one multi-center cohort study focused on Chinese PAD patients who had MetS. In addition, compared with other Chinese research studies, this cohort appears to have the longest follow-up times and largest sample size.

\section{Conclusion}

We observed that that the prevalence of PAD in Chinese MetS patients was $24.4 \%$. A Cox regression model analysis revealed that an abnormal ABI value was an independent, inversely factor correlated with the all-cause mortality $(\mathrm{RR}=$ 1.82, 95\% CI: $1.45-2.34, \mathrm{p}<0.01)$ and cardiovascular disease-related mortality $(\mathrm{RR}=1.88,95 \% \mathrm{CI}: 1.51-2.90, \mathrm{p}<$ 0.01). Our study demonstrated that an abnormal ABI value was not only a significant and independent risk factor, but also was an independent predictor of CVD and a decrease in the survival rate in hospitalized Chinese patients with MetS. Routine evaluation of the ABI could be helpful for identifying high risk patients, especially among MetS patients.

\section{The authors state that they have no Conflict of Interest (COI).}

Funding: The study was sponsored by the Shanghai Educational Development Foundation (11ZR1428300).

Contributors: Yan Cang and Jue Li had full access to all of the data in the study and take responsibility for the integrity of the data and the accuracy of the data analysis. Study concept and design: Yan Cang, Jue Li. Acquisition of data: Yan Cang, Jue Li, Yun Zhou, Yuanmin Li, Yuezhou Wu, Xiankai Li, Chaofan Wang, Haiyan Yang, Yawei Xu. Analysis and interpretation of data: Yan Cang, Jue Li. Drafting of the manuscript: Yan Cang, Jue Li. Critical revision of the manuscript for important intellectual content: Yan Cang, Jue Li. Statistical analysis: Yan Cang, Jue Li. Study supervision: Yan Cang, Jue Li.

Yan Cang and Jue Li contributed equally to this work. 


\section{References}

1. Nenci GG, et al. Unifying concept of arterial vascular disease. Eur Heart J 1A: A27-A30, 1999.

2. Safar ME, Frohlich ED. The arterial system in hypertension. Hypertension J 26: 10-14, 1995.

3. Rahn KH, Barenbrock M, Hausberg M, Kosch M, Suwelack B, Witta J. Vessel wall alterations in patients with renal failure. Hypertens Res 23: 3-6, 2000.

4. Newman AB. Peripheral arterial disease: insights from population studies of older adults. J Am Geriatr Soc 48: 1157-1162, 2000.

5. Zheng ZJ, Sharrett AR, Chambless LE, et al. Association of anklebrachial index with clinical coronary heart disease, stroke and preclinical carotid and potential atherosclerosis: the Atherosclerosis Risk in Communities (ARIC) Study. Atherosclerosis 131: 115-125, 1997.

6. Dieter RS, Chu WW, Pacanowski JP, et al. The significance of lower extremity peripheral disease. Clin Cardiol 25: 3-10, 2002.

7. Hirsch AT, Haskal ZJ, Hertzer NR, et al. ACC/AHA 2005 practice guidelines for the management of patients with peripheral arterial disease. Circulation 113: e463-e465, 2006.

8. Ouriel K. Peripheral arterial disease. Lancet 358: 1257-1264, 2001.

9. Allison MA, Hiatt WR, Hirsch AT, Coll JR, Criqui MH. A high ankle-brachial index is associated with increased cardiovascular disease morbidity and lower quality of life. J Am Coll Cardiol 51: 1292-1298, 2008

10. Criqui MH, McClelland RL, McDermott MM, et al. The anklebrachial index and incident cardiovascular events in the MESA (Multi-Ethnic Study of Atherosclerosis). J Am Coll Cardiol 56: 1506-1512, 2010.

11. Hooi JD, Kester AD, Stoffers HE, et al. Asymptomatic peripheral arterial occlusive disease predicted cardiovascular morbidity and mortality in a 7-year follow-up study. J Clin Epidemiol 57: 294300, 2004.

12. Otah KE, Madan A, Otah E, et al. Usefulness of an abnormal ankle-brachial index to predict presence of coronary artery disease in African-Americans. Am J Cardiol 93: 481-483, 2004.

13. Timothy PM, Murphy TP, Michael J, et al. Ankle-brachial index and cardiovascular risk prediction: an analysis of 11,594 individuals with 10-year follow-up. Atherosclerosis 10: 037, 2011.

14. Victor A, Michael H, Julie O, et al. Risk factors for progression of peripheral arterial disease in large and small vessels. Circulation 113: 2623-2629, 2006.

15. Heather L, Gornik J, Joshua A. Peripheral Arterial Disease (PAD). Circulation 111: e169-e172, 2005.

16. Fletcher B, Berra K, Lynne $\mathrm{T}$, et al. Managing abnormal blood lipids. Circulation 112: 3184-3209, 2005.

17. Brevetti G, Laurenzano E, Giugliano G, et al. Metabolic syndrome and cardiovascular risk prediction in peripheral arterial disease. Nutr Metab Cardiovasc Dis 20: 676-682, 2010.

18. Rosnick HE, Lindsay RS, Mc Dermott MM, et al. Relationship of high and low ankle brachial index to all-cause and cardiovascular disease mortality. The strong heart study. Circulation 109: 733739, 2004.

19. Heagerty PJ, Lumley T, Pepe MS. Time-dependent ROC curves for censored survival data and a diagnostic marker. Biometrics 56: 337-344, 2000.

20. Brindle P, Emberson J, Lampe F, et al. Predictive accuracy of the Framingham coronary risk score in British men: prospective cohort study. BMJ 327: 1267, 2003.

21. Fabsitz RR, Sidawy AN, Go O, Lee ET, Welty TK, Devereux RB. Prevalence of peripheral arterial disease and associates risk factors in American Indians: the strong heart study. Am J Epidemiol 149: 330-338, 1999.

22. The American College of Cardiology foundation and the American
Heart Association. ACC/AHA guidelines for the management of patients with peripheral arterial disease. Circulation 113: 14741547, 2006.

23. Hiatt WR, Marshall JA, Baxter J, et al. Diagnostic methods for peripheral arterial disease in the San Luis Valley Diabetes Study. J Clin Epidemiol 43: 597-606, 1990.

24. World Health Organization. Definition, Diagnosis and Classification of Diabetes Mellitus and its Complications. Report of a WHO Consultation. Part 1: Diagnosis and Classification of Diabetes and Mellitus. Geneva, World Health Organization 1999.

25. The DECODA Study Group. Prevalence of the metabolic syndrome in populations of Asian origin Comparison of the IDF definition with the NCEP definition. Diabet Res Clin Pract 76: 57-67, 2007.

26. Jian-jun Wang, Hong-bing Li, Kinnunen L, et al. How well does the metabolic syndrome defined by five definitions predict incident diabetes and incident coronary heart disease in a Chinese population? Atherosclerosis 192: 161-168, 2007.

27. Executive summary of the third report of The National Cholesterol Education Program (NCEP) Expert Panel on detection, evaluation, and treatment of high blood cholesterol in adults. Adult Treatment Panel III. JAMA 285: 2486-2497, 2001.

28. Anon. The Atherosclerosis Risk in Communities (ARIC) Study. design and objectives. The ARIC investigators. Am J Epidemiol 129: 687-702, 1989.

29. Rose G, McCartney P, Reid DD. Self-administration of a questionnaire on chest pain and intermittent claudication. Br J Prev Soc Med 31: 42-48, 1977.

30. Fowkes FG, Murray GD, Butcher I, et al; Ankle Brachial Index Collaboration. Ankle brachial index combined with Framingham Risk Score to predict cardiovascular events and mortality: a metaanalysis. JAMA 300: 197-208, 2008.

31. Summary of the third report of the national cholesterol education program (NCEP) expert panel on detection, evaluation, and treatment of high blood cholesterol in adults (Adult Treatment Panel III). Circulation 10: 227-239, 2004.

32. Hirsch AT, Haskal ZJ, Hertzer NR, et al. ACC/AHA 2005 practice guidelines for the management of patients with peripheral arterial disease (lower extremity, renal, mesenteric, and abdominal aortic): a collaborative report from the American Association for Vascular Surgery/Society for Vascular Surgery Society for Cardiovascular Angiography and Interventions, Society for Vascular Medicine and Biology, Society of Interventional Radiology, and the ACC/AHA Task Force on Practice Guidelines (Writing Committee to Develop Guidelines for the Management of Patients With Peripheral Arterial Disease): endorsed by the American Association of Cardiovascular and Pulmonary Rehabilitation; National Heart, Lung, and Blood Institute; Society for Vascular Nursing; TransAtlantic InterSociety Consensus; and Vascular Disease Foundation. Circulation 113: e463-e654, 2006.

33. Norgren L, Hiatt WR, Dormandy JA, et al. Inter-society consensus for the management of peripheral arterial disease (TASC II). Eur J Vasc Endovasc Surg 33(Suppl 1): S1-S75, 2007.

34. Graham I, Atar D, Borch-Johnsen K, et al. European guidelines on cardiovascular disease prevention in clinical practice: full text Fourth Joint Task Force of the European Society of Cardiology and other societies on cardiovascular disease prevention in clinical practice (constituted by representatives of nine societies and by invited experts). Eur J Cardiovasc Prev Rehabil 14(Suppl 2): S1S113, 2007.

35. Beckman JA, Jaff MR, Creager MA. The United States preventive services task force recommendation statement on screening for peripheral arterial disease: more harm than benefit? Circulation 114: 861-866, 2006.

36. Murphy TP, Dhangana R, Pencina MJ, Zafar AM, D'Agostino RB. Performance of current guidelines for coronary heart disease 
prevention: optimal use of the Framingham-based risk assessment. Atherosclerosis 216: 452-457, 2011.

37. Beckman JA, Creager MA, Libby P. Diabetes and atherosclerosis. Epidemiology, pathophysiology and management. JAMA 287: 2570-2581, 2002.

38. Ridker PM, Brown NJ, Vaughan DE, Harrison DG, Mehta JL. Established and emerging plasma biomarkers in the prediction of first atherothrombotic events. Circulation 109: IV6-IV19, 2004.

39. Dhangana R, Murphy TP, Pencina MJ, Zafar AM. Prevalence of low ankle-brachial index elevated plasma fibrinogen and CRP across Framingham risk categories: data from the National Health and Nutrition Examination Survey (NHANES) 1999-2004. Atherosclerosis 216: 174-179, 2011.

(C) 2012 The Japanese Society of Internal Medicine http://www.naika.or.jp/imonline/index.html 